\title{
Geometric Methods for Invariant Zero Cancellation in Discrete-Time Non-strictly-proper Linear Multivariable Systems
}

\author{
G. Marro and E. Zattoni
}

\begin{abstract}
This paper presents a geometric procedure for designing a minimal-order dynamic feedforward compensator whose aim is cancelling the minimum-phase invariant zeros of a discrete-time linear multivariable system, non-strictly proper in general. The feedforward compensator also satisfies the condition of being of minimal dynamic order and that of maintaining right invertibility: i.e., if the original system is right invertible, then the cascade of the feedforward compensator and the system is right invertible as well. Special attention is paid to this property since it is a basic property in interesting control problems, like, e.g., reference tracking. Nonetheless, the procedure is developed for non-right-invertible and non-leftinvertible systems, in general.
\end{abstract}

\section{INTRODUCTION}

Zero cancellation is a problem widely discussed in system and control theory. In fact, it can be approached under various perspectives and with different further specifications.

A method for cancellation of simple invariant zeros in linear multivariable systems, is introduced in [1]. The limitation on the multiplicity of the invariant zeros is basically due to the fact that the algorithm presented relies on the so-called invariant-zero directions, which are related to eigenvector problems in the presence of multiple eigenvalues.

In [2], the problem of zero displacement is treated from an essentially polynomial perspective. The problem is stated in a very general context, where the rational matrix functions considered are not necessarily proper. Further requirements of the solutions may be those of having minimum McMillan degree or being $J$-unitary and $J$-inner, either with respect to the imaginary axis or to the unit circle.

The design procedure for a precompensator cancelling the invariant zeros of a strictly-proper continuous-time system is set forth in [3]. The theoretical developments are based on the extensive use of the special coordinate basis, which enables dealing with cancellation of multiple invariant zeros, while maintaining relevant properties of the original system like stabilizability, right-invertibility and left-invertibility. However, the compensator thus obtained is not necessarily the compensator with the minimal dynamic order.

In this work, zero cancellation in discrete-time linear multivariable systems is encompassed in the framework of the geometric approach (see, e.g., [4], [5]). The systems addressed are not required to be either right-invertible or leftinvertible. However, the feedforward compensator guarantees right-invertibility of the cascade if the original system is right invertible. Nonetheless, if left-invertibility must be

The authors are with the Department of Electronics, Computer Science, and Systems, University of Bologna, 40136 Bologna, Italy. Email: giovanni.marro and elena. zattoni@unibo.it maintained, a filter to be connected in cascade with the original system and such that to cancel its minimum-phase invariant zeros, while preserving left-invertibility, can be obtained by means of simple duality arguments.

The determination of an appropriate state feedback as a friend of the maximal output-nulling controlled invariant subspace, with the additional requirement of assigning the internal eigenvalues of the constrained reachability subspace in such a way that their set be disjoint from that of the system invariant zeros, is the first and most important step of a procedure aimed at pointing out a key geometric subspace and correlated matrices that capture the structure of the invariant zeros to be cancelled. In fact, the subsequent steps consists of a sequence of similarity transformations whose goal is to lead to the coordinates where a basis matrix of the resolving subspace can easily be determined. Then, the crucial matrices associated to it are found as the solution of a pair of algebraic equations which are the Sylvester equation and the output-nulling equation. Those matrices will be the dynamic matrix and the output distribution matrix of the feedforward compensator, which therefore, has the property of being the feedforward compensator with the minimal dynamic order.

Notation: $\mathbb{R}$ stands for the set of real numbers. $\mathbb{C}^{\odot}, \mathbb{C}^{\otimes}$, and $\mathbb{C}^{\circ}$ respectively stand for the open unit disc, the open set outside the unit disc, and the unit circle. Matrices and linear maps are denoted by capital letters, like $A$. The spectrum, the image, and the kernel of $A$ are denoted by $\sigma(A), \operatorname{im} A$, and ker $A$, respectively. The restriction of a linear map $A$ to an $A$ invariant subspace $\mathcal{J}$ is denoted by $\left.A\right|_{\mathcal{J}}$. The quotient space of a vector space $\mathcal{X}$ over a subspace $\mathcal{V} \subseteq \mathcal{X}$ is denoted by $\mathcal{X} / \mathcal{V}$. The dimension of $\mathcal{V}$ is denoted by $\operatorname{dim} \mathcal{V}$. The symbols $I_{n}$ and $O_{m \times n}$ are respectively used for the identity matrix of dimension $n$ and the $m \times n$ zero matrix (subscripts are omitted when the dimensions are clear from the context).

\section{Geometric ApProAch BACKGROUND}

The discrete-time linear time-invariant system

$$
\begin{aligned}
x_{t+1} & =A x_{t}+B u_{t}, \\
y_{t} & =C x_{t}+D u_{t},
\end{aligned}
$$

is considered, where $t \in \mathbb{Z}$ is the time variable and $x \in$ $\mathcal{X}=\mathbb{R}^{n}, u \in \mathbb{R}^{p}, y \in \mathbb{R}^{q}$, with $p \leq n$ and $q \leq n$, are the state, the input, the output, respectively. $A, B, C$, $D$ are constant real matrices of appropriate dimensions. $\left[\begin{array}{l}B \\ D\end{array}\right]$ and $\left[\begin{array}{ll}C & D\end{array}\right]$ are full-rank matrices. Geometric objects extensively used in this work are $\mathcal{B}$, the image of $B, \mathcal{C}$, 
the kernel of $C, \min \mathcal{J}(A, \mathcal{B})$, the minimal $A$-invariant subspace containing $\mathcal{B}, \max \mathcal{J}(A, \mathcal{C})$, the maximal $A$-invariant subspace contained in $\mathcal{C}, \mathcal{V}^{*}=\max \mathcal{V}(A, B, C, D)$, the maximal output-nulling controlled invariant subspace of (1), (2), $\mathcal{S}^{*}=\min \mathcal{S}(A, B, C, D)$, the minimal input-containing conditioned invariant subspace of (1), (2), $\mathcal{R}_{\mathcal{V}^{*}}=\mathcal{V}^{*} \cap \mathcal{S}^{*}$, the reachability subspace on $\mathcal{V}^{*}$. The following geometric properties are extensively used in this work. A subspace $\mathcal{V} \subseteq \mathcal{X}$ is an output-nulling controlled invariant subspace of (1), (2) if and only if at least one linear map $F$ exists, such that $(A+B F) \mathcal{V} \subseteq \mathcal{V}$ and $\mathcal{V} \subseteq \operatorname{ker}(C+D F)$. A subspace $\mathcal{S} \subseteq \mathcal{X}$ is an input-containing conditioned invariant subspace of (1), (2) if and only if at least one linear map $G$ exists, such that $(A+G C) \mathcal{S} \subseteq \mathcal{S}$ and $\mathcal{S} \supseteq \operatorname{im}(B+G D)$. Let the linear map $F$ be such that $(A+B F) \mathcal{V}^{*} \subseteq \mathcal{V}^{*}$ and $\mathcal{V}^{*} \subseteq \operatorname{ker}(C+D F)$, then $(A+B F) \mathcal{R}_{\mathcal{V}^{*}} \subseteq \mathcal{R}_{\mathcal{V}^{*}}$ and $\mathcal{R}_{\mathcal{V}^{*}} \subseteq \operatorname{ker}(C+D F)$ hold with the same $F$. The spectrum of $\left.(A+B F)\right|_{\mathcal{R}_{\mathcal{V}^{*}}}$ is assignable. The spectrum of $(A+$ $B F)\left.\right|_{\mathcal{V}^{*} / \mathcal{R}_{\mathcal{V}^{*}}}$ is fixed. The spetrum of $\left.(A+B F)\right|_{\mathcal{V}^{*} / \mathcal{R}_{\mathcal{V}^{*}}}$ is also known as the set of the internal unassignable eigenvalues of $\mathcal{V}^{*}$ or, equivalently, as the set of the invariant zeros of (1), (2), and is also denoted by $\mathcal{Z}(A, B, C, D)$. With a slight abuse of terminology, the invariant zeros of (1), (2) lying in $\mathbb{C}^{\odot}$ are called the minimum-phase invariant zeros of (1), (2) and their set is denoted by $\mathcal{Z}_{M P}(A, B, C, D)$. Similarly, the invariant zeros of (1), (2) lying in $\mathbb{C}^{\otimes}$ are called the nonminimum-phase invariant zeros of (1), (2) and their set is denoted by $\mathcal{Z}_{N M P}(A, B, C, D)$. The set of the invariant zeros of (1), (2) lying in $\mathbb{C}^{\circ}$ is denoted by $\mathcal{Z}_{0}(A, B, C, D)$. Further crucial notions are those of right-invertibility and left invertibility of a system. A geometric condition equivalent to the property of system (1), (2) of being right-invertible is $\mathcal{V}^{*}+\mathcal{S}^{*}=\mathcal{X}$. A geometric condition equivalent to the property of system (1), (2) of being left-invertible is $\mathcal{V}^{*} \cap \mathcal{S}^{*}=\{0\}$.

\section{Problem Statement}

Consider system (1), (2) and assume that $\mathcal{R}=$ $\min \mathcal{J}(A, \mathcal{B})=\mathcal{X}$, where $\mathcal{R}$ is the reachable subspace of $(A, B)$, and $\mathcal{Q}=\max \mathcal{J}(A, \mathcal{C})=\{0\}$, where $\mathcal{Q}$ is the unobservable subspace of $(A, C)$, so that (1), (2) be a minimal state-space description. Consider the problem of designing a feedforward compensator,

$$
\begin{aligned}
x_{c, t+1} & =A_{c} x_{c, t}+B_{c} v_{t}, \\
u_{t} & =C_{c} x_{c, t}+D_{c} v_{t},
\end{aligned}
$$

such that the cascade of (3), (4) and the original system (1), (2) has a minimal state-space description

$$
\begin{aligned}
x_{m, t+1} & =A_{m} x_{m, t}+B_{m} v_{t}, \\
y_{t} & =C_{m} x_{m, t}+D_{m} v_{t},
\end{aligned}
$$

that satisfies the following conditions:

$\mathcal{C} 1$. the invariant zeros are the nonminimum-phase invariant zeros and the invariant zeros on the unit circle of the original system: i.e.,

$$
\begin{aligned}
& \mathcal{Z}\left(A_{m}, B_{m}, C_{m}, D_{m}\right)= \\
& \quad \mathcal{Z}_{N M P}(A, B, C, D) \cup \mathcal{Z}_{0}(A, B, C, D) ;
\end{aligned}
$$

$\mathcal{C} 2$. is right-invertible if (1), (2) is right-invertible.

\section{SyNTHESIS OF THE FEEDFORWARD COMPENSATOR}

This section is aimed at revealing the structure of the minimum-phase invariant zeros of the original system, so that it can be repeated in the feedforward compensator. This is achieved by identifying a specific output-nulling controlled invariant subspace of the original system, henceforth denoted by $\mathcal{V}_{m}$, whose internal eigenvalues matches the set of the minimum-phase invariant zeros of the original system.

Lemma 1: Consider system (1), (2). Let the linear map $F$ be such that $(A+B F) \mathcal{V}^{*} \subseteq \mathcal{V}^{*}$ and $\mathcal{V}^{*} \subseteq \operatorname{ker}(C+D F)$. Perform the similarity transformation $T=\left[\begin{array}{llll}T_{1} & T_{2} & T_{3} & T_{4}\end{array}\right]$, where $\operatorname{im} T_{1}=\mathcal{R}_{\mathcal{V}^{*}}$, im $\left[T_{1} T_{2}\right]=\mathcal{V}^{*}$, im $\left[T_{1} T_{3}\right]=\mathcal{S}^{*}$. Then, $A_{F}^{\prime}=T^{-1}(A+B F) T=\left[\begin{array}{cccc}A_{11}^{\prime} & A_{12}^{\prime} & A_{13}^{\prime} & A_{14}^{\prime} \\ O & A_{22}^{\prime} & A_{23}^{\prime} & A_{24}^{\prime} \\ O & O & A_{33}^{\prime} & A_{34}^{\prime} \\ O & O & A_{43}^{\prime} & A_{44}^{\prime}\end{array}\right],(7)$
$C_{F}^{\prime}=(C+D F) T=\left[\begin{array}{cccc}O & O & C_{3}^{\prime} & C_{4}^{\prime}\end{array}\right]$.

Proof: Let $n_{1}=\operatorname{dim} \mathcal{R}_{\mathcal{V}^{*}}, n_{2}=\operatorname{dim} \mathcal{V}^{*}-n_{1}, n_{3}=$ $\operatorname{dim} \mathcal{S}^{*}-n_{1}$, and $n_{4}=n-n_{1}-n_{2}-n_{3}$. Let

$$
\begin{aligned}
T_{1}^{\prime} & =\left[\begin{array}{llll}
I_{n_{1}} & O & O & O
\end{array}\right]^{\top} \\
T_{2}^{\prime} & =\left[\begin{array}{llll}
O & I_{n_{2}} & O & O
\end{array}\right]^{\top} \\
T_{3}^{\prime} & =\left[\begin{array}{llll}
O & O & I_{n_{3}} & O
\end{array}\right]^{\top} \\
T_{4}^{\prime} & =\left[\begin{array}{llll}
O & O & O & I_{n_{4}}
\end{array}\right]^{\top} .
\end{aligned}
$$

With respect to the new coordinates, $\mathcal{R}_{\mathcal{V}^{*}}=\operatorname{im} T_{1}^{\prime}, \mathcal{V}^{*}=$

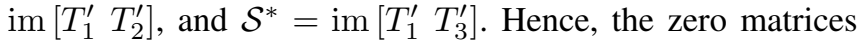
in the first block of columns of $A_{F}^{\prime}$ are due to $(A+B F)$ invariance of $\mathcal{R}_{\mathcal{V}^{*}}$. The zero matrices in the last blocks of rows of $A_{F}^{\prime}$ are due to $(A+B F)$-invariance of $\mathcal{V}^{*}$. The zero matrices in $C_{F}^{\prime}$ are due to $\mathcal{R}_{\mathcal{V}^{*}} \subseteq \mathcal{V}^{*} \subseteq \operatorname{ker}(C+D F)$.

Remark 1: The set of the internal eigenvalues of $\mathcal{R}_{\mathcal{V}^{*}}$ is equal to the set of the eigenvalues of $A_{11}^{\prime}$ : i.e., $\sigma((A+$ $\left.B F)\left.\right|_{\mathcal{R}_{\mathcal{V}^{*}}}\right)=\sigma\left(A_{11}^{\prime}\right)$. The set of the internal unassignable eigenvalues of $\mathcal{V}^{*}$, or, equivalently, the set of the invariant zeros of (1), (2), is equal to the set of the eigenvalues of $A_{22}^{\prime}$ : i.e., $\sigma\left(\left.(A+B F)\right|_{\mathcal{V}^{*} / \mathcal{R}_{\mathcal{V}^{*}}}\right)=\mathcal{Z}(A, B, C, D)=\sigma\left(A_{22}^{\prime}\right)$.

Lemma 2: Consider system (1), (2). Let the linear map $F$ be such that $(A+B F) \mathcal{V}^{*} \subseteq \mathcal{V}^{*}, \mathcal{V}^{*} \subseteq \operatorname{ker}(C+D F)$, and $\sigma\left(\left.(A+B F)\right|_{\mathcal{R}_{\mathcal{V}^{*}}}\right) \cap \sigma\left(\left.(A+B F)\right|_{\mathcal{V}^{*} / \mathcal{R}_{\mathcal{V}^{*}}}\right)=\emptyset$. Refer to (7), (8) and perform the similarity transformation $T^{\prime}=$ $\left[T_{1}^{\prime} T_{2}^{\prime \prime} T_{3}^{\prime} T_{4}^{\prime}\right]$, where $T_{1}^{\prime}, T_{3}^{\prime}, T_{4}^{\prime}$ were defined in (9), (11), (12), and $T_{2}^{\prime \prime}=\left[\begin{array}{llll}X^{\top} & I_{n_{2}} & O & O\end{array}\right]^{\top}$, with $X$ denoting the solution of the Sylvester equation

$$
A_{11}^{\prime} X-X A_{22}^{\prime}=-A_{12}^{\prime} .
$$


Then,

$$
\begin{aligned}
& A_{F}^{\prime \prime}=T^{\prime-1} A_{F}^{\prime} T^{\prime}=\left[\begin{array}{cccc}
A_{11}^{\prime} & O & A_{13}^{\prime \prime} & A_{14}^{\prime \prime} \\
O & A_{22}^{\prime} & A_{23}^{\prime} & A_{24}^{\prime} \\
O & O & A_{33}^{\prime} & A_{34}^{\prime} \\
O & O & A_{43}^{\prime} & A_{44}^{\prime}
\end{array}\right], \\
& C_{F}^{\prime \prime}=C_{F}^{\prime} T^{\prime}=C_{F}^{\prime} .
\end{aligned}
$$

Proof: In light of Remark 1, the assumptions on $F$ ensure the existence and uniqueness of the solution of (13). Hence, (14), (15) follow from (7), (8), since

$$
T^{\prime-1}=\left[\begin{array}{cccc}
I_{n_{1}} & -X & O & O \\
O & I_{n_{2}} & O & O \\
O & O & I_{n_{3}} & O \\
O & O & O & I_{n_{4}}
\end{array}\right]
$$

Lemma 3: Consider system (1), (2). Let the linear map $F$ be such that $(A+B F) \mathcal{V}^{*} \subseteq \mathcal{V}^{*}, \mathcal{V}^{*} \subseteq \operatorname{ker}(C+D F)$, and $\sigma\left(\left.(A+B F)\right|_{\mathcal{R}_{\mathcal{V}^{*}}}\right) \cap \sigma\left(\left.(A+B F)\right|_{\mathcal{V}^{*} / \mathcal{R}_{\mathcal{V}^{*}}}\right)=\emptyset$. Refer to (14), (15). Let $\mathcal{J}_{s}=\operatorname{im} J_{s}$ be the maximal $A_{22}^{\prime}$-invariant subspace such that $\sigma\left(A_{22}^{\prime} \mid \mathcal{J}_{s}\right) \subset \mathbb{C}^{\odot}$. Let $\mathcal{J}_{0}=\operatorname{im} J_{0}$ be the maximal $A_{22}^{\prime}$-invariant subspace such that $\sigma\left(A_{22}^{\prime} \mid \mathcal{J}_{0}\right) \subset \mathbb{C}^{\circ}$. Let $\mathcal{J}_{u}=\operatorname{im} J_{u}$ be the maximal $A_{22}^{\prime}$-invariant subspace such that $\sigma\left(\left.A_{22}^{\prime}\right|_{\mathcal{J}_{u}}\right) \subset \mathbb{C}^{\otimes}$. Let $J_{s}, J_{0}$, and $J_{u}$ be full-rank matrices. Perform the similarity transformation $T^{\prime \prime}=\left[\begin{array}{lll}T_{1}^{\prime} T_{2}^{\prime \prime \prime} T_{3}^{\prime} T_{4}^{\prime}\end{array}\right]$, where $T_{1}^{\prime}, T_{3}^{\prime}, T_{4}^{\prime}$ were defined by (9), (11), (12), and $T_{2}^{\prime \prime \prime}=\left[\begin{array}{llll}O & J^{\top} & O & O\end{array}\right]^{\top}$, with $J=\left[\begin{array}{lll}J_{s} & J_{0} & J_{u}\end{array}\right]$. Then,

$$
\begin{aligned}
& A_{F}^{\prime \prime \prime}=T^{\prime \prime-1} A_{F}^{\prime \prime} T^{\prime \prime}=\left[\begin{array}{cccc}
A_{11}^{\prime} & O & A_{13}^{\prime \prime} & A_{14}^{\prime \prime} \\
O & A_{22}^{\prime \prime} & A_{23}^{\prime \prime} & A_{24}^{\prime \prime} \\
O & O & A_{33}^{\prime} & A_{34}^{\prime} \\
O & O & A_{43}^{\prime} & A_{44}^{\prime}
\end{array}\right], \\
& C_{F}^{\prime \prime \prime}=C_{F}^{\prime \prime} T^{\prime \prime}=C_{F}^{\prime},
\end{aligned}
$$

with

$$
A_{22}^{\prime \prime}=\left[\begin{array}{ccc}
A_{22_{s}}^{\prime \prime} & O & O \\
O & A_{22_{0}}^{\prime \prime} & O \\
O & O & A_{22_{u}}^{\prime \prime}
\end{array}\right] .
$$

Proof: Equations (16), (17) follow from (14), (15), since since $J$ is invertible by construction and, therefore,

$$
T^{\prime \prime-1}=\left[\begin{array}{cccc}
I_{n_{1}} & O & O & O \\
O & J^{-1} & O & O \\
O & O & I_{n_{3}} & O \\
O & O & O & I_{n_{4}}
\end{array}\right]
$$

Moreover, in the new coordinates,

$\mathcal{J}_{s}=\operatorname{im}\left[\begin{array}{c}I_{n_{s}} \\ O \\ O\end{array}\right], \mathcal{J}_{0}=\operatorname{im}\left[\begin{array}{c}O \\ I_{n_{0}} \\ O\end{array}\right], \mathcal{J}_{u}=\operatorname{im}\left[\begin{array}{c}O \\ O \\ I_{n_{u}}\end{array}\right]$,

with $n_{s}=\operatorname{dim} \mathcal{J}_{s}, n_{0}=\operatorname{dim} \mathcal{J}_{0}$, and $n_{u}=\operatorname{dim} \mathcal{J}_{u}$. Hence, the zero matrices in $A_{22}^{\prime \prime}$ are due to $A_{22}^{\prime}$-invariance of $\mathcal{J}_{s}$, $\mathcal{J}_{0}$, and $\mathcal{J}_{u}$.

Theorem 1: Consider system (1), (2). Let the linear map $F$ be such that $(A+B F) \mathcal{V}^{*} \subseteq \mathcal{V}^{*}, \mathcal{V}^{*} \subseteq \operatorname{ker}(C+D F)$, and $\sigma\left(\left.(A+B F)\right|_{\mathcal{R}_{\mathcal{V}^{*}}}\right) \cap \sigma\left(\left.(A+B F)\right|_{\mathcal{V}^{*} / \mathcal{R}_{\mathcal{V}^{*}}}\right)=\emptyset$. Refer to the following more detailed representation of (16), (17) with (18)

$$
A_{F}^{\prime \prime \prime}=\left[\begin{array}{cccccc}
A_{11}^{\prime} & O & O & O & A_{13}^{\prime \prime} & A_{14}^{\prime \prime} \\
O & A_{22_{s}}^{\prime \prime} & O & O & A_{23_{s}}^{\prime \prime} & A_{24_{s}}^{\prime \prime} \\
O & O & A_{22_{0}}^{\prime \prime} & O & A_{23_{0}}^{\prime \prime} & A_{24_{0}}^{\prime \prime} \\
O & O & O & A_{22_{u}}^{\prime \prime} & A_{23_{u}}^{\prime \prime} & A_{24_{u}}^{\prime \prime} \\
O & O & O & O & A_{33}^{\prime} & A_{34}^{\prime} \\
O & O & O & O & A_{43}^{\prime} & A_{44}^{\prime}
\end{array}\right],
$$

$C_{F}^{\prime \prime \prime}=\left[\begin{array}{llllll}O & O & O & O & C_{3}^{\prime} & C_{4}^{\prime}\end{array}\right]$.

With respect to these coordinates, let $\mathcal{V}_{m}$ be defined by

$$
\mathcal{V}_{m}=\operatorname{im} V_{m}^{\prime \prime \prime}=\operatorname{im}\left[\begin{array}{llllll}
O & I_{n_{s}} & O & O & O & O
\end{array}\right]^{\top} \text {. }
$$

Then,

(i) $\mathcal{V}_{m}$ is an output-nulling controlled invariant subspace of (1), (2);

(ii) the internal eigenvalues of $\mathcal{V}_{m}$ are the minimum-phase invariant zeros of (1), (2): i.e., $\sigma\left((A+B F) \mid \mathcal{V}_{m}\right)=$ $\mathcal{Z}_{M P}(A, B, C, D)$.

Proof: Proposition (i): Equation

$$
A_{F}^{\prime \prime \prime} V_{m}^{\prime \prime \prime}=V_{m}^{\prime \prime \prime} A_{22_{s}}^{\prime \prime}
$$

holds, owing to (19) and (21). Hence, $\mathcal{V}_{m}$ is an $(A+B F)$ invariant subspace or, equivalently, an $(A, \mathcal{B})$-controlled invariant subspace. Moreover,

$$
C_{F}^{\prime \prime \prime} V_{m}^{\prime \prime \prime}=O
$$

holds, owing to (20) and (21). Hence, $\mathcal{V}_{m}$ is an output-nulling controlled invariant subspace.

Proposition (ii) follows from Proposition (i) since, in light of Remark 1, (22) implies $\sigma\left(\left.(A+B F)\right|_{\mathcal{V}_{m}}\right)=\sigma\left(A_{22_{s}}^{\prime \prime}\right)=$ $\mathcal{Z}_{M P}(A, B, C, D)$.

Corollary 1: Consider system (1), (2). Let the linear map $F$ be such that $(A+B F) \mathcal{V}^{*} \subseteq \mathcal{V}^{*}, \mathcal{V}^{*} \subseteq \operatorname{ker}(C+D F)$, and $\sigma\left(\left.(A+B F)\right|_{\mathcal{R}_{\mathcal{V}^{*}}}\right) \cap \sigma\left(\left.(A+B F)\right|_{\mathcal{V}^{*} / \mathcal{R}_{\mathcal{V}^{*}}}\right)=\emptyset$. Let $T_{m}=$ $T T^{\prime} T^{\prime \prime}$, where $T, T^{\prime}, T^{\prime \prime}$ were introduced in Lemmas 1-3. Refer to (21) and let $V_{m}=T_{m} V_{m}^{\prime \prime \prime}$. Then,

$$
\begin{aligned}
A V_{m}-V_{m} W & =-B L, \\
C V_{m} & =-D L,
\end{aligned}
$$

hold with $W=A_{22_{s}}^{\prime \prime}$ and $L=F V_{m}$.

Proof: Owing to (7), (8), (14)-(17) and the definitions of $T_{m}$ and $V_{m}, A_{F}^{\prime \prime \prime}=T_{m}^{-1}(A+B F) T_{m}, C_{F}^{\prime \prime \prime}=(C+$ $D F) T_{m}$, and $V_{m}^{\prime \prime \prime}=T_{m}^{-1} V_{m}$. Consequently, (22) and (23) can be written as $T_{m}^{-1}(A+B F) V_{m}=T_{m}^{-1} V_{m} A_{22_{S}}^{\prime \prime}$ and $(C+D F) V_{m}^{*}=O$. Then, one gets (24) and (25) by left multiplying the former by $T_{m}$ and replacing $A_{22_{S}}^{\prime \prime}$ and $F V_{m}$ with $W$ and $L$, respectively.

Corollary 1 , which refers to a basis matrix $V_{m}$ of $\mathcal{V}_{m}$ in the original coordinates, has provided a consistent pair of matrices, $W$ and $L$, which point out the structure of the internal eigenvalues of $\mathcal{V}_{m}$, or equivalently, the structure of the minimum-phase invariant zeros of the original system. The matrices $W$ and $L$ will respectively be the system matrix and the output distribution matrix of the feedforward compensator. The input distribution matrix and the 
direct feedthrough matrix of the feedforward compensator will respectively be such that the states of the feedforward compensator and the inputs of the original system be directly accessible one by one. Hence, the feedforward compensator is described by system (3), (4) with

$$
\begin{aligned}
& A_{c}=W, \quad B_{c}=\left[\begin{array}{ll}
I_{n_{s}} & O
\end{array}\right], \\
& C_{c}=L, \quad D_{c}=\left[\begin{array}{ll}
O & I_{p}
\end{array}\right] .
\end{aligned}
$$

The compensator has the minimal dynamic order since the system matrix exactly duplicates the dynamics of the minimum-phase zeros of the original system.

\section{Properties of the Cascade}

This section examines the properties of the cascade of the feedforward compensator and the original system. First, a minimal form of the series of the feedforward compensator and the original system will be obtained. Then, it will be shown that the conditions of the problem statement are satisfied. The series of the feedforward compensator (3), (4), with (26), (27), and the original system (1), (2) is ruled by

$$
\begin{aligned}
x_{s, t+1} & =A_{s} x_{s, t}+B_{s} v_{t}, \\
y_{t} & =C_{s} x_{s, t}+D_{s} v_{t},
\end{aligned}
$$

where

$$
\begin{aligned}
& A_{s}=\left[\begin{array}{cc}
A & B C_{c} \\
O & A_{c}
\end{array}\right], \quad B_{s}=\left[\begin{array}{c}
B D_{c} \\
B_{c}
\end{array}\right], \\
& C_{s}=\left[\begin{array}{ll}
C & D C_{c}
\end{array}\right], \quad D_{s}=D D_{c} .
\end{aligned}
$$

Theorem 2, that follows, introduces a minimal representation of the series (28), (29), with (30), (31). Lemma 4 sets the basis for the similarity transformation used to prove Theorem 2.

Lemma 4: Consider system (28), (29), with (30), (31). Let

$$
\begin{aligned}
& \mathcal{J}=\operatorname{im} J=\operatorname{im}\left[\begin{array}{cc}
I_{n} & O
\end{array}\right]^{\top}, \\
& \mathcal{J}_{c}=\operatorname{im} J_{c}=\operatorname{im}\left[\begin{array}{ll}
V_{m}^{\top} & I_{n_{s}}
\end{array}\right]^{\top} .
\end{aligned}
$$

Then,

(i) $\mathcal{J}$ is an $A_{s}$-invariant subspace;

(ii) $\mathcal{J}_{c}$ is an $A_{s}$-invariant subspace;

(iii) $\mathcal{J} \oplus \mathcal{J}_{c}=\mathcal{X}_{s}$, where $\oplus$ stands for the direct sum of subspaces and $\mathcal{X}_{s}$ denotes the state space of (28), (29).

Proof: Proposition (i) is implied by $A_{s} J=J A$, which holds by virtue of (30) and (32).

Proposition (ii) is implied by $A_{s} J_{c}=J_{c} A_{s}$, which holds by virtue of (30) and (33), in light of (24), (26), and (27).

Proposition (iii) follows from the comparison of the respective basis matrices $J$ and $J_{c}$ of $\mathcal{J}$ and $\mathcal{J}_{c}$, defined by (32) and (33).

Theorem 2: Consider system (5), (6). Let

$$
\begin{array}{ll}
A_{m}=A, & B_{m}=\left[\begin{array}{ll}
-V_{m} & B
\end{array}\right], \\
C_{m}=C, & D_{m}=\left[\begin{array}{ll}
O & D
\end{array}\right] .
\end{array}
$$

Then, system (5), (6) with (34), (35) is a minimal state-space representation of (28), (29) with (30), (31).
Proof: Let $T_{s}=\left[\begin{array}{ll}J & J_{c}\end{array}\right]$, where $J$ and $J_{c}$ were defined in (32) and (33). Owing to Proposition (iii) of Lemma 4, $T_{s}$ is invertible. Hence, let the similarity transformation $T_{s}$ be applied to (28), (29) with (30), (31). Then,

$$
\begin{aligned}
& A_{s}^{\prime}=T_{s}^{-1} A_{s} T_{s}=\left[\begin{array}{cc}
A & O \\
O & A_{c}
\end{array}\right], \\
& B_{s}^{\prime}=T_{s}^{-1} B_{s}=\left[\begin{array}{cc}
-V_{m} & B \\
I & O
\end{array}\right], \\
& C_{s}^{\prime}=C_{s} T_{s}=\left[\begin{array}{ll}
C & O
\end{array}\right], \\
& D_{s}^{\prime}=D_{s}=\left[\begin{array}{ll}
O & D
\end{array}\right],
\end{aligned}
$$

where (24)-(27) have been taken into account. Then, one gets (34), (35) from (36)-(39) by dropping the subsystem described by the triple $\left(A_{c}, I, O\right)$, which is unobservable. Moreover, from (34), (35) it follows that (5), (6) is reachable and observable, since (1), (2) is reachable and observable by assumption. Therefore, (5), (6) with (34), (35) is a minimal state-space representation of (28), (29) with (30), (31).

In the remainder of this section, it will be shown that system (5), (6), with (34), (35), satisfies Conditions $\mathcal{C} 1$ and $\mathcal{C} 2$ (Theorems 3 and 4 , respectively). The proofs involve a close comparison between the basic subspaces of the original system and those of the minimal form of the cascade. This is carried out in Lemmas 5-7, with an extensive use of the geometric tools reviewed in Appendix I.

Lemma 5: Consider system (1), (2) and system (5), (6), with (34), (35). Let $\mathcal{V}^{*}=\max \mathcal{V}(A, B, C, D)$ and $\mathcal{V}_{m}^{*}=$ $\max \mathcal{V}\left(A_{m}, B_{m}, C_{m}, D_{m}\right)$. Then,

$$
\mathcal{V}_{m}^{*}=\mathcal{V}^{*}
$$

Proof: As reviewed in Appendix I (Proposition 1), a basis matrix of $\mathcal{V}^{*}=\max \mathcal{V}(A, B, C, D)$ can be derived from that of $\hat{\mathcal{V}}^{*}=\max \mathcal{V}(\hat{A}, \hat{B}, \hat{C})$, where the matrices of the extended triple $(\hat{A}, \hat{B}, \hat{C})$ are defined by (51). Similarly, a basis matrix of $\mathcal{V}_{m}^{*}=\max \mathcal{V}\left(A_{m}, B_{m}, C_{m}, D_{m}\right)$ can be derived from that of $\hat{\mathcal{V}}_{m}^{*}=\max \mathcal{V}\left(\hat{A}_{m}, \hat{B}_{m}, \hat{C}_{m}\right)$, where the matrices of the extended triple $\left(\hat{A}_{m}, \hat{B}_{m}, \hat{C}_{m}\right)$ are defined according to (51), with $A, B, C, D$ respectively replaced by $A_{m}, B_{m}, C_{m}, D_{m}$. Owing to (34), (35), $\hat{A}_{m}=\hat{A}$,

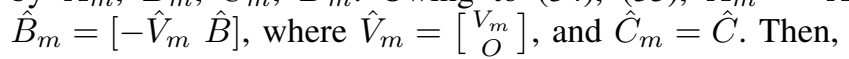

$$
\begin{aligned}
\hat{\mathcal{V}}_{m}^{*} & =\max \mathcal{V}\left(\hat{A}_{m}, \hat{B}_{m}, \hat{C}_{m}\right) \\
& =\max \mathcal{V}\left(\hat{A},\left[-\hat{V}_{m} \hat{B}\right], \hat{C}\right) \\
& =\max \mathcal{V}(\hat{A}, \hat{B}, \hat{C})=\hat{\mathcal{V}}^{*},
\end{aligned}
$$

since, as is shown in [5] (Property 4.2-1), for any subspace $\hat{\mathcal{H}}=\operatorname{im} \hat{H} \subseteq \hat{\mathcal{V}}^{*}$, the relation $\max \mathcal{V}(\hat{A},[\hat{B} \hat{H}], \hat{C})=$ $\max \mathcal{V}(\hat{A}, \hat{B}, \hat{\bar{C}})=\hat{\mathcal{V}}^{*}$ holds and $\hat{\mathcal{V}}_{m}=\operatorname{im} \hat{V}_{m} \subseteq \hat{\mathcal{V}}^{*}$ holds by construction. Therefore, (40) follows from (41) and Proposition 1.

Lemma 6: Consider system (1), (2) and system (5), (6), with (34), (35). Let $\mathcal{S}^{*}=\min \mathcal{S}(A, B, C, D)$ and $\mathcal{S}_{m}^{*}=$ $\min \mathcal{S}\left(A_{m}, B_{m}, C_{m}, D_{m}\right)$. Then,

$$
\mathcal{S}_{m}^{*}=\mathcal{S}^{*}+\mathcal{V}_{m}
$$

Proof: As reviewed in Appendix I (Proposition 2), a basis matrix of $\mathcal{S}^{*}=\min \mathcal{S}(A, B, C, D)$ can be derived 
from that of $\hat{\mathcal{S}}^{*}=\min \mathcal{S}(\hat{A}, \hat{B}, \hat{C})$, where the matrices of the extended triple $(\hat{A}, \hat{B}, \hat{C})$ are defined by (53). Similarly, a basis matrix of $\mathcal{S}_{m}^{*}=\min \mathcal{S}\left(A_{m}, B_{m}, C_{m}, D_{m}\right)$ can be derived from that of $\hat{\mathcal{S}}_{m}^{*}=\min \mathcal{S}\left(\hat{A}_{m}, \hat{B}_{m}, \hat{C}_{m}\right)$, where the matrices of the extended triple $\left(\hat{A}_{m}, \hat{B}_{m}, \hat{C}_{m}\right)$ are defined according to (53), with $A, B, C, D$ respectively replaced by $A_{m}, B_{m}, C_{m}, D_{m}$. Owing to (34), (35), $\hat{A}_{m}, \hat{B}_{m}, \hat{C}_{m}$ have the structure

$$
\begin{aligned}
\hat{A}_{m} & =\left[\begin{array}{ccc}
A & -V_{m} & B \\
O & O & O \\
O & O & O
\end{array}\right], \quad \hat{B}_{m}=\left[\begin{array}{cc}
O & O \\
I_{n_{s}} & O \\
O & I_{p}
\end{array}\right], \\
\hat{C}_{m} & =\left[\begin{array}{lll}
C & O & D
\end{array}\right] .
\end{aligned}
$$

Hence, by applying Algorithm 2 to the extended triple $\left(\hat{A}_{m}, \hat{B}_{m}, \hat{C}_{m}\right)$ and taking into account Proposition 2, one gets

$\hat{\mathcal{S}}_{m}^{*}=\operatorname{im}\left[\begin{array}{cccc}S^{*} & V_{m} & O & O \\ O & O & I_{n_{s}} & O \\ O & O & O & I_{p}\end{array}\right]=\operatorname{im}\left[\begin{array}{ccc}S_{m}^{*} & O & O \\ O & I_{n_{s}} & O \\ O & O & I_{p}\end{array}\right]$,

where $S^{*}$ and $V_{m}$ are basis matrices of $\mathcal{S}^{*}$ and $\mathcal{V}_{m}$, respectively. Hence, (43) implies $\mathcal{S}_{m}^{*}=\operatorname{im} S_{m}^{*}=\operatorname{im}\left[S^{*} V_{m}\right]=$ $\mathcal{S}^{*}+\mathcal{V}_{m}$, which proves (42).

Lemma 7: Consider system (1), (2) and system (5), (6), with (34), (35). Let $\mathcal{R}_{\mathcal{V}^{*}}=\mathcal{V}^{*} \cap \mathcal{S}^{*}$ and $\mathcal{R}_{\mathcal{V}_{m}^{*}}=\mathcal{V}_{m}^{*} \cap \mathcal{S}_{m}^{*}$. Then,

$$
\mathcal{R}_{\mathcal{V}_{m}^{*}}=\mathcal{R}_{\mathcal{V}^{*}}+\mathcal{V}_{m}
$$

Proof: Owing to Lemmas 5, 6, and the inclusion $\mathcal{V}_{m} \subseteq$ $\mathcal{V}^{*}$, the relation $\mathcal{R}_{\mathcal{V}_{m}^{*}}=\mathcal{V}_{m}^{*} \cap \mathcal{S}_{m}^{*}=\mathcal{V}^{*} \cap\left(\mathcal{S}^{*}+\mathcal{V}_{m}\right)=$ $\mathcal{V}^{*} \cap \mathcal{S}^{*}+\mathcal{V}_{m}=\mathcal{R}_{\mathcal{V}^{*}}+\mathcal{V}_{m}$ holds, which implies (44).

Theorem 3: Consider system (1), (2) and system (5), (6), with (34), (35). Let $\mathcal{Z}_{N M P}(A, B, C, D)$ be the set of the nonminimum-phase invariant zeros of (1), (2). Let $\mathcal{Z}_{0}(A, B, C, D)$ be the set of the invariant zeros of (1), (2) on the unit circle $\mathbb{C}^{\circ}$. Let $\mathcal{Z}\left(A_{m}, B_{m}, C_{m}, D_{m}\right)$ be the set of the invariant zeros of (5), (6). Then,

$$
\begin{aligned}
& \mathcal{Z}\left(A_{m}, B_{m}, C_{m}, D_{m}\right)= \\
& \quad \mathcal{Z}_{N M P}(A, B, C, D) \cup \mathcal{Z}_{0}(A, B, C, D) .
\end{aligned}
$$

Proof: Let $F_{m}$ be such that $\left(A_{m}+B_{m} F_{m}\right) \mathcal{V}_{m}^{*} \subseteq \mathcal{V}_{m}^{*}$ and $\mathcal{V}_{m}^{*} \subseteq \operatorname{ker}\left(C_{m}+D_{m} F_{m}\right)$. Then,

$$
\mathcal{Z}\left(A_{m}, B_{m}, C_{m}, D_{m}\right)=\sigma\left(\left.\left(A_{m}+B_{m} F_{m}\right)\right|_{\mathcal{V}_{m}^{*} / \mathcal{R}_{\mathcal{V}_{m}^{*}}}\right)
$$

and is independent of $F_{m}$. Let $F$ be such that $(A+B F) \mathcal{V}^{*} \subseteq$ $\mathcal{V}^{*}$ and $\mathcal{V}^{*} \subseteq \operatorname{ker}(C+D F)$. Then,

$$
\begin{array}{r}
\mathcal{Z}_{N M P}(A, B, C, D) \cup \mathcal{Z}_{0}(A, B, C, D)= \\
\sigma\left(\left.(A+B F)\right|_{\mathcal{V}^{*} /\left(\mathcal{R}_{\mathcal{V}^{*}}+\mathcal{V}_{m}\right)}\right)
\end{array}
$$

and is independent of $F$. Therefore, (45) follows from the comparison of (46) with (47), in light of (34), (35), Lemma 5, and Lemma 7.

Theorem 4: Consider system (1), (2) and system (5), (6), with (34), (35). If (1), (2) is right-invertible, then, (5), (6) is right-invertible.
Proof: Owing to Lemma 5, Lemma 6, the inclusion $\mathcal{V}_{m} \subseteq \mathcal{V}^{*}$, and the assumption of right-invertibility of (1), (2) the following relation holds:

$$
\mathcal{V}_{m}^{*}+\mathcal{S}_{m}^{*}=\mathcal{V}^{*}+\mathcal{S}^{*}+\mathcal{V}_{m}=\mathcal{V}^{*}+\mathcal{S}^{*}=\mathcal{X}=\mathbb{R}^{n},
$$

which implies right-invertibility of (5), (6).

A slight modification of the procedure leads to a filter cancelling a subset of the minimum-phase zeros of the original system - even only one zero with its multiplicity. More precisely, the basis transformation $T^{\prime \prime}$, considered in Lemma 3, must be defined in such a way that matrix $A_{22}^{\prime \prime}$ turns out to be in Jordan form. Then, the definition of the key subspace $\mathcal{V}_{m}$, given in Theorem 1, must be modified so that the basis matrix $V_{m}^{\prime \prime \prime}$ have an identity matrix that selects the Jordan blocks of $A_{22}^{\prime \prime}$ corresponding to the minimum-phase zeros to be cancelled. Still, $\mathcal{V}_{m}$ is an output nulling controlled invariant subspace of (1), (2) and Corollary 1 points out the structure of the minimum-phase invariant zeros to be cancelled.

The dynamic system for zero cancellation can alternatively be derived in the form of a filter to be connected in cascade of the original system by means of simple duality arguments. In that case, the property of the original system which is preserved along with reachability and observability is leftinvertibility. While preserving right-invertibility is relevant in control problems like, e.g., reference tracking, preserving left-invertibility is important in observation problems, like, e.g., input reconstruction.

\section{CONCLUSION}

In this paper, zero cancellation in discrete-time linear multivariable systems is discussed in the framework of the geometric approach. The systems addressed are not necessarily assumed to be either right-invertible or leftinvertible. Nonetheless, how to preserve these properties has been shown. The procedure is based on the determination of a key subspace and an associated pair of matrices that capture the structure of the invariant zeros to be cancelled. The feedforward compensator consistently designed has the minimal dynamic order.

\section{APPENDIX I \\ GEOMETRIC ALgORITHMS FOR NON-STRICTLY-PROPER SYSTEMS}

Some geometric properties of non-strictly-proper systems can be studied by reducing them to strictly-proper systems, according to techniques mentioned, e.g., in [5] and derived from [6]. This section reviews how the maximal outputnulling controlled invariant subspace and the minimal inputcontaining conditioned invariant subspace can be respectively obtained by means of the algorithms for computing the maximal controlled invariant subspace contained in the kernel of the output and the minimal conditioned invariant subspace containing the image of the input, which are the basic subspaces of strictly-proper systems. 
The discrete-time linear time-invariant system

$$
\begin{aligned}
\hat{x}_{t+1} & =\hat{A} \hat{x}_{t}+\hat{B} u_{t}, \\
y_{t} & =\hat{C} \hat{x}_{t},
\end{aligned}
$$

is considered, where $t \in \mathbb{Z}$ is the time variable and $\hat{x} \in$ $\hat{\mathcal{X}}=\mathbb{R}^{\hat{n}}, u \in \mathbb{R}^{p}, y \in \mathbb{R}^{q}$, with $p \leq \hat{n}$ and $q \leq \hat{n}$, are the state, the input, the output, respectively. $\hat{A}, \hat{B}, \hat{C}$ are constant real matrices of appropriate dimensions. $\hat{B}$ and $\hat{C}$ are full-rank matrices. $\hat{\mathcal{B}}$ and $\hat{\mathcal{C}}$ stand for $\operatorname{im} \hat{B}$ and $\operatorname{ker} \hat{C}$, respectively. The symbol $\hat{\mathcal{V}}^{*}=\max \mathcal{V}(\hat{A}, \hat{B}, \hat{C})$ stands for the maximal $(\hat{A}, \hat{\mathcal{B}})$-controlled invariant subspace contained in $\hat{\mathcal{C}}$. The symbol $\hat{\mathcal{S}}^{*}=\min \mathcal{S}(\hat{A}, \hat{B}, \hat{C})$ stands for the minimal $(\hat{A}, \hat{\mathcal{C}})$-conditioned invariant subspace containing $\hat{\mathcal{B}}$. A subspace $\hat{\mathcal{V}} \subseteq \hat{\mathcal{X}}$ is an $(\hat{A}, \hat{\mathcal{B}})$-controlled invariant subspace if and only if at least one linear map $\hat{F}$ exists, such that $(\hat{A}+\hat{B} \hat{F}) \hat{\mathcal{V}} \subseteq \hat{\mathcal{V}}$. A subspace $\hat{\mathcal{S}} \subseteq \hat{\mathcal{X}}$ is an $(\hat{A}, \hat{\mathcal{C}})$-conditioned invariant subspace if and only if at least one linear map $\hat{G}$ exists, such that $(\hat{A}+\hat{G} \hat{C}) \hat{\mathcal{S}} \subseteq \hat{\mathcal{S}}$. The subspaces $\hat{\mathcal{V}}^{*}$ and $\hat{\mathcal{S}}^{*}$ are computed with the following algorithms.

Algorithm 1: Consider system (49), (50). The subspace $\hat{\mathcal{V}}^{*}$ is the last term of the sequence

$$
\begin{aligned}
& \hat{\mathcal{V}}_{0}=\hat{\mathcal{C}} \\
& \hat{\mathcal{V}}_{i}=\hat{A}^{-1}\left(\hat{\mathcal{V}}_{i-1}+\hat{\mathcal{B}}\right) \cap \hat{\mathcal{C}}, \quad i=1,2, \ldots, k,
\end{aligned}
$$

where $k<\hat{n}$ is the least integer such that $\hat{\mathcal{V}}_{k+1}=\hat{\mathcal{V}}_{k}$.

Algorithm 2: Consider system (49), (50). The subspace $\hat{\mathcal{S}}^{*}$ is the last term of the sequence

$$
\begin{aligned}
& \hat{\mathcal{S}}_{0}=\hat{\mathcal{B}} \\
& \hat{\mathcal{S}}_{i}=\hat{A}\left(\hat{\mathcal{S}}_{i-1} \cap \hat{\mathcal{C}}\right)+\hat{\mathcal{B}}, \quad i=1,2, \ldots, k,
\end{aligned}
$$

where $k<\hat{n}$ is the least integer such that $\hat{\mathcal{S}}_{k+1}=\hat{\mathcal{S}}_{k}$.

The following propositions relate the basic subspaces of a quadruple, like (1), (2), to the corresponding subspaces of triples, like (49), (50), conveniently constructed. Proofs will be omitted for the sake of brevity.
Proposition 1: Consider system (1), (2). Let $\mathcal{V}^{*}=$ $\max \mathcal{V}(A, B, C, D)$. Consider system (49), (50), with

$$
\hat{A}=\left[\begin{array}{cc}
A & O \\
C & O
\end{array}\right], \hat{B}=\left[\begin{array}{c}
B \\
D
\end{array}\right], \hat{C}=\left[\begin{array}{ll}
O & I_{q}
\end{array}\right] .
$$

Let $\hat{\mathcal{V}}^{*}=\max \mathcal{V}(\hat{A}, \hat{B}, \hat{C})$. Then,

$$
\hat{\mathcal{V}}^{*}=\operatorname{im} \hat{V}^{*}=\operatorname{im}\left[\begin{array}{c}
V^{*} \\
O
\end{array}\right],
$$

where $V^{*}$ is a basis matrix of $\mathcal{V}^{*}$.

In light of Proposition 1 , the computation of $\mathcal{V}^{*}=$ $\max \mathcal{V}(A, B, C, D)$ reduces to applying Algorithm 1 to the system (49), (50), with $\hat{A}, \hat{B}, \hat{C}$ defined by (51).

Proposition 2: Consider system (1), (2). Let $\mathcal{S}^{*}=$ $\min \mathcal{S}(A, B, C, D)$. Consider system (49), (50), with

$$
\hat{A}=\left[\begin{array}{cc}
A & B \\
O & O
\end{array}\right], \hat{B}=\left[\begin{array}{c}
O \\
I_{p}
\end{array}\right], \hat{C}=\left[\begin{array}{ll}
C & D
\end{array}\right] .
$$

Let $\hat{\mathcal{S}}^{*}=\min \mathcal{S}(\hat{A}, \hat{B}, \hat{C})$. Then,

$$
\hat{\mathcal{S}}^{*}=\operatorname{im} \hat{S}^{*}=\operatorname{im}\left[\begin{array}{cc}
S^{*} & O \\
O & I_{p}
\end{array}\right],
$$

where $S^{*}$ is a basis matrix of $\mathcal{S}^{*}$

In light of Proposition 2, the computation of $\mathcal{S}^{*}=$ $\min \mathcal{S}(A, B, C, D)$ reduces to applying Algorithm 2 to the system (49), (50), with $\hat{A}, \hat{B}, \hat{C}$ defined by (53).

\section{REFERENCES}

[1] J. Douglas and M. Athans, "Multivariable poles, zeros, and pole/zero cancellation," in The Control Handbook, W. S. Levine, Ed. Boca Raton, Florida: CRC Press, 1996, pp. 445-450.

[2] C. Oară and R. Andrei, "Zero cancellation for general rational matrix functions," Linear Algebra and its Applications, vol. 431, pp. 20002023, 2009.

[3] Y. Wan, S. Roy, and A. Saberi, "Explicit precompensator design for invariant-zero cancellation," International Journal of Control, vol. 82 , no. 5, pp. 808-811, May 2009.

[4] W. M. Wonham, Linear Multivariable Control: A Geometric Approach, 3rd ed. New York: Springer-Verlag, 1985.

[5] G. Basile and G. Marro, Controlled and Conditioned Invariants in Linear System Theory. Englewood Cliffs, New Jersey: Prentice Hall, 1992 .

[6] B. D. O. Anderson, "Output-nulling invariant and controllability subspaces," in Proceedings of the 6th IFAC World Congress, Boston/Cambridge, MA, USA, August 24-30, 1975, Paper 46.3. 